\title{
La píldora con 20 ug de Etinilestradiol y 75 ug de gestodeno Una nueva opción en anticoncepción oral de microdosis
}

\author{
Jaime Urdinola MD*
}

\begin{abstract}
RESUMEN
La píldora con 20 ug de Etinilestradiol (EE) y 75 ug de Gestodeno (GTD) aparece en 1998 como una nueva alternativa en Colombia para la anticoncepción, con una clara disminución de sus efectos secundarios y como consecuencia con una mejor tolerancia, sin perder Ia seguridad anticonceptiva y manteniendo adecuado control del ciclo. Es un paso adelante en el progreso y desarrollo de los Anticonceptivos Orales (AO), bienvenido no sólo por los médicos sino por sus pacientes.
\end{abstract}

PALABRAS CLAVES: Anticonceptivos orales, etinil estradiol 20 ug, gestodeno 75 ug.

\section{SUMMARY}

The contraceptive pill with 20 ug ethynilestradiol (EE) and 75 ug gestodene (GTD) appears in Colombia in 1998 as a new alternative, clearly with a reduction of its secondary effects and therefore with better tolerance, without loosing its contraceptive efficacy and mantaining a proper cycle control. It represents a step forwards in the development of oral contraceptives, wellcome not only by physicians but also by their patients.

KEY WORDS: Oral contraceptives, 20 ug ethynilestradiol, 75 ug Gestodene.

\section{Introducción}

Los anticonceptivos orales (AO) han sido usados por 40 años en el mundo, calculándose actualmente que hay 63 millones de mujeres usuarias, 8.3 millones en América Latina y aproximadamente 1 millón en Colombia (1). Por esta razón, cualquier variación en su composición o en la dosis de los AO tiene un impacto considerable (2).

Los AO han experimentado en este sentido múltiples modificaciones y mejoras, producto de las experiencias obtenidas y de la investigación realizada. Las píldoras actuales son por lo tanto muy diferentes de los primeros compuestos de hace casi cuatro décadas.

En 1957 apareció en el mercado Enovid (B) de Searle, píldora combinada de $9.7 \mathrm{mg}$ de noretinodrel y $150 \mathrm{ug}$ de mestranol para el manejo de hemorragias uterinas de origen disfuncional, aprobada en 1959 por la Administración Federal de Drogas de Estados Unidos (FDA) como AO. En Alemania apareció en junio de 1961 el Anovlar $21 \circledR$, combinación de 50 ug de EE y $4 \mathrm{mg}$ de acetato de noretisterona (NETA) (3). Inman y Vessey demostraron claramente en 1968 (4) que el número de casos con embolia pulmonar y de trombosis cerebrales se había aumentado en las usuarias de $\mathrm{AO}>50$ ug de etinilestradiol (EE), de 2 a 8 veces más que en las no usuarias.

Los resultados de la investigación anterior condujeron a la aparición de los preparados de microdosis $<$ de 50

\footnotetext{
* Profesor de la Universidad El Bosque. Director Unidad de Clima-
} terio y Menopausia. Hospital Simón Bolívar de Santafé de Bogotá. ug de EE desde 1973, para disminuir el riesgo cerebro y cardiovascular.

\section{AO con 20 ug de EE}

Loestrin $1 / 20 ®$ fue el primer preparado diseñado para reducir la incidencia de efectos colaterales asociados con los estrógenos, como los eventos tromboembólicos. Su tasa informada de embarazo fue de 0.98 por 100 años mujer, en 12.241 ciclos de 1336 mujeres, así como 35\% de hemorragia uterina irrregular en el primer ciclo y de 15\% después de 15 meses de uso. No existen estudios comparativos actualizados con la metodología que se emplea hoy en día, aunque la presentación se sigue comercializando en USA.

En 1987 aparece en el mercado un $\mathrm{AO}$ de 20 ug que incorpora uno de los nuevos gestágenos de tercera generación, el desogestrel (150 ug DSG) (Lovelleß). En 1997 son aprobados en USA (Alesse ${ }^{\circledR}$ ) y en Alemania (Leios ${ }^{\circledR}$ / Miranova( ) AO también con 20 ug de EE y 100 ug de levonorgestrel (LNG) (Tabla 1).

Durante este año de 1998 se introduce en el mercado colombiano el más nuevo de los $\mathrm{AO}$ con otro gestágeno de 3a. generación: Harmonet $₫ /$ Femiane $₫$ cuya composición incorpora el gestodeno (GTD) (Tabla 2).

\section{Razones para la reducción del EE a 20 ug en los AO}

Dentro de las ventajas, se presentará menor incidencia de los eventos relacionados con la dosis del estrógeno, esperando que la seguridad metabólica a largo plazo llegue a ser mayor que con los anteriores $\mathrm{AO}$. 
Tabla 1

NUEVOS ANTICONCEPTIVOS ORALES CON 20 UG EE

\begin{tabular}{|c|c|c|c|}
\hline Nombre & ug Gestágeno & Compañía & País \\
\hline Lovelle® / Mercilon® & 150 DSG & Organon & Europa/Colombia \\
\hline Alesse® / Leios® & $100 \mathrm{LNG}$ & Wyeth & USA/Alemania. \\
\hline Miranova® & $100 \mathrm{LNG}$ & Schering $A G$ & Alemania \\
\hline Femiane® & 75 GTD & Wyeth & Colombia \\
\hline
\end{tabular}

Tabla 2

TIPOS DE AO Y SUS MODIFICACIONES EN EL TIEMPO

\begin{tabular}{|c|c|c|}
\hline Tipo de Píldora & Características & $\begin{array}{l}\text { Lugar/Año } \\
\text { de introducción }\end{array}$ \\
\hline Macrodosis & $\begin{array}{l}\text { Enovid } \AA=150 \text { ug Mestranol }+9.7 \mathrm{mg} \text { Noretinodrel } \\
\text { Anovlar } 21{ }^{\circledR}=50 \mathrm{ug} \text { EE }+4 \mathrm{mg} \text { NETA }\end{array}$ & $\begin{array}{l}\text { USA/1959 } \\
\text { Alemania/1961 }\end{array}$ \\
\hline Microdosis & Microgynon $® /$ Nordette ${ }^{\circledR}=30$ ug EE +150 ug LNG & Alemania/1973 \\
\hline Trifásicas & $\begin{array}{l}\text { Triquilar® / Triphasil@ }=30 / 40 / 30 \text { ug EE }+ \\
\text { 50/75/125 ug LNG }\end{array}$ & Alemania/1979 \\
\hline Con nuevos gestágenos & $\begin{array}{l}\text { Marvelon } \AA=30 \text { ug EE }+150 \text { ug DSG } \\
\text { Gynovin } ® / \text { Minulet } \AA=30 \text { ug EE }+75 \text { ug GTD } \\
\text { Cilest }{ }^{\circledR}=35 \text { ug EE }+240 \text { ug NGT }\end{array}$ & $\begin{array}{l}\text { Holanda/1981 } \\
\text { Alemania/USA/1986 } \\
\text { USA /1987 }\end{array}$ \\
\hline 20 ug EE & $\begin{array}{l}\text { Loestrin } 1 / 20 \AA=1 \text { mg NETA } \\
\text { Mercilon } \AA=150^{\circ} \text { ug DSG } \\
\text { Harmonet } \AA / \text { Femiane } \AA\end{array}$ & $\begin{array}{l}\text { USA/1973 } \\
\text { Holanda/1989 } \\
\text { USA/Alemania/1997 }\end{array}$ \\
\hline
\end{tabular}

$\mathrm{EE}=$ etinilestradiol. $\mathrm{NETA}=$ acetato de noretindrona. $\mathrm{LNG}=$ levonorgestrel. $\mathrm{DSG}=$ desogestrel. $\mathrm{GTD}=$ gestodeno NGT $=$ norgestimato.

Como desventajas teóricas podría disminuirse la eficacia anticonceptiva comparativa con los otros AO, obtener un pobre control del ciclo y posible pérdida de beneficios no anticonceptivos, como la protección documentada contra el carcinoma de ovario o endometrio.

De acuerdo con los datos que se presentan más adelante, la eficacia anticonceptiva con este $\mathrm{AO}$ no se compromete y el control del ciclo es adecuado y la tolerancia es buena.

La protección para el carcinoma de ovario o endometrio no se reduce con este $\mathrm{AO}$ de 20 ug de EE, ya que este es un beneficio no anticonceptivo dependiente del gestágeno -en este caso del GTD- que sigue conservando sus dosis comprobadamente anovulatoria per-se de $75 \mathrm{ug}$, presente en el otro preparado tradicional con 30 ug de EE (Minulet(B/ Gynovin(B) (5-6).

El riesgo para posibles complicaciones tromboembólicas -el cual se explica más adelante- es tan bajo, que no tiene mayor impacto en la decisión de aceptación de los AO por parte de la paciente. En cambio, cerca del $50 \%$ de las mujeres experimentan algunos cambios como aumento transitorio del peso, cefalea, cambios en el humor y mastalgia, atribuidos a los estrógenos y responsables de la mitad de las razones para descontinuar los AO $(7,10)$ (Tabla 3).

Descontinuar los AO puede llevar a un embarazo no planeado. Si reducir la cantidad del estrógeno puede ayudar a minimizar los efectos adversos, la aparición de los AO con 20 ug de estrógeno será un paso importante en el desarrollo de la anticoncepción oral.

\section{Riesgo de Enfermedad Tromboembólica Venosa con el uso de los AO}

Gerstman (8) ha demostrado cómo se reduce el riesgo para enfermedad tromboembólica venosa sólo con disminuir la dosis del estrógeno en los AO (Gráfica 1). Este resultado se obtiene sin tener en cuenta el papel de la mutación del Factor $\mathrm{V}$ de Leiden, el cual eleva el riesgo en forma considerable (9) (Tabla 4).

Tabla 3

INCIDENCIA DE EFECTOS ADVERSOS EN 19095 CICLOS DE 670 MUJERES DURANTE 36 MESES

\begin{tabular}{|c|c|c|c|}
\hline & $\mathbf{n}$ & $\%$ & \\
\hline \multicolumn{2}{|c|}{ Uno o más efectos adversos } & 192 & \multirow[t]{7}{*}{28.7} \\
\hline Cefalea & 105 & 15.7 & \\
\hline Mastalgia & 56 & 8.4 & \\
\hline Migraña & 36 & 5.4 & \\
\hline Nerviosidad & 28 & 4.2 & \\
\hline Náusea & 25 & 3.7 & \\
\hline Ref 17 & & & \\
\hline
\end{tabular}


Tabla 4

INCIDENCIA DE TROMBOSIS VENOSA X 10.000 AÑOS/MUJER Y EL FACTOR V DE LEIDEN

- Mujeres sin este defecto y no usuarias de AO 0.8

- Mujeres de 15 a 49 años en general

- Mujeres en general y que toman AO

2.1

- Mujeres portadoras de la mutación del factor V

- Mujeres portadoras y usuarias de AO

- Embolia pulmonar mortal como complicación

más severa de la mujer joven

28.5

- 400.00 mil mujeres jóvenes examinadas para

evitar una muerte por embolia pulmonar

$\mathrm{AO}=$ Anticonceptivos Orales - Ref. 9

Tabla 5

\section{EMBARAZOS}

20 ug EE /75 ug GTD ( $\mathrm{n}=218 / 2495$ ciclos)

- Embarazo debido a falla del método

- Embarazo debido a error en la toma

- Embarazo no relacionado con el estudio

20 ug EE /150 ug DSG ( $\mathbf{n}=219 / 2496$ ciclos)

- Embarazo debido a falla del método

20 ug EE /75 ug GTD ( $\mathrm{n}=218 / 2495$ ciclos $)$

- Embarazo debido a error en la toma

$\mathrm{EE}=$ etinilestradiol $\mathrm{GTD}=$ gestodeno $\mathrm{DSG}=$ desogestrel Ref. 22.

A la Hipoproacelerenimia o Parahemofilia A es a lo que conlleva la mutación del Factor $\mathrm{V}$ de Leiden o de Lovaina, Bélgica, el factor de riesgo genético más frecuente para la trombosis venosa profunda, la cual puede presentarse hasta en un $5 \%$ de la población general. El riesgo se verá aumentado de manera significativa en aquellas mujeres con el defecto y que a la vez toman AO. Es muy importante por lo tanto, que aún sin disponer de la prueba de laboratorio que determina la mutación, se haga énfasis en el interrogatorio minucioso acerca del antecedente de los problemas tromboembólicos personales o en la familia, antes de la prescripción de los AO. Este sencillo elemento ha sido de gran valor para identificar pacientes provenientes de familias con tendencia a la trombosis venosa múltiple.

\section{Problemas Potenciales con la Reducción de la Dosis en los AO a 20 ug de EE}

La inhibición de la ovulación es la principal acción terapéutica de los AO. Esta puede perderse si hay una reducción exagerada de la dosis de los estrógenos, pero no sucede con los AO de 20 ug de EE.

La acción supresora de la función ovárica obtenida por la inhibición del eje hipotálamo-hipófisis-ovario, depende tanto de la dosis administrada de EE como de la del gestágeno y de su estructura química. Si no hay inhibición, el resultado será la maduración folicular, la formación de los denominados quistes ováricos que en este caso no deben identificarse como patológicos, y la probable aparición de una ovulación inesperada con los síntomas clínicos de hemorragia por disrupción y dolor pélvico así como mastalgia.

Mall-Haefeli estudió 71 mujeres divididas en 2 grupos durante 8 ciclos, 6 de tratamiento y 1 antes y después del mismo con la píldora con el gestágeno DSG. 38 recibieron el AO con 20 ug EE y 33 el de 30 ug EE. Los valores de FSH no fueron totalmente suprimidos con el $\mathrm{AO}$ con 20 ug de EE, lo que si ocurrió con el de 30 ug EE en forma significativa, aunque el estradiol y la progesterona séricas sí disminuyeron significativamente con ambos preparados (10).

Es muy importante también considerar que la seguridad anticonceptiva no sea afectada al disminuir la dosis del EE, aún en el caso de que el cumplimiento sea pobre. Otra investigación sobre la inhibición de la ovulación con dos $\mathrm{AO}$ que contenían 20 ug de EE reveló algún desarrollo folicular con ambos preparados, pero sin evidencia de ovulación (11). Y otro estudio que comparaba la inhibición de la ovulación con $3 \mathrm{AO}$, una preparación monofásica con 30 ug de EE, una preparación trifásica conteniendo 30 - 40 ug de EE y un preparado monofásico de 20 ug de EE, informó similares hallazgos a los antes anotados (12). Es decir, que no se presentó la ovulación, aunque hubo mayor desarrollo folicular con el preparado de 20 ug, y la ovulación por escape que ocurrió se presentó en el preparado monofásico de 30 ug de EE por pobre cumplimiento, al omitir tres píldoras al comienzo del ciclo. Es prudente -de todas maneras- considerar que la información clínica disponible es aún limitada y que se necesita de los estudios a largo plazo que confirmen estos alentadores datos iniciales (13).

Otro argumento de peso relacionado con la disminución de la dosis del estrógeno en los AO sería el que podrían disminuirse los beneficios no anticonceptivos de los preparados combinados. En este caso los factores que más afectarían a la población sería el de la disminución en la incidencia de cáncer endometrial y ovárico. Y aunque la población podría ser afectada, es muy bajo el número de usuarias conscientes acerca de estos beneficios. La protección obtenida en contra del cáncer endometrial es mayor en las mujeres con baja paridad, el mayor número de las que se atienden actualmente. El estudio CASH (14) muestra un riesgo relativo (RR) promedio de 0.2 para la nulípara que toma la píldora por 5 años o más, manteniéndose dicha protección hasta 10 años después de descontinuarla.

Pero debe recordarse que los gestágenos son los que se indican en el tratamiento del cáncer endometrial, ya que este es un efecto progestacional domostrado. Este efecto no se verá por lo tanto afectado en los $\mathrm{AO}$ con 20 ug de EE, ya que depende del gestágeno y no del estrógeno.

La protección contra el cáncer ovárico lleva a una reducción del $40 \%$ en el riesgo después de 5 años de usar los $\mathrm{AO}$, manteniéndose también por lo menos hasta 10 años después de descontinuarlos. La evidencia epidemiológica sugiere que la protección es conferida por la acción de inhibición de la ovulación, no esperán- 
dose tampoco que la reducción en la dosis estrogénica a $20 \mathrm{ug}$ afecte esto (15).

Hay una publicación conflictiva sobre un estudio prospectivo a 5 años de mujeres que tomaron la píldora con 20 ug EE combinada con DSG comparadas con las que no recibieron tratamiento hormonal (16), sugiriendo los autores que la píldora no le permite a las mujeres jóvenes desarrollar al máximo su densidad ósea. Sinembargo hay que anotar que las diferencias fueron muy pequeñas, y que estos hallazgos entran en conflicto con la investigación realizada sobre la terapia hormonal de sustitución en el climaterio, la que ha domostrado que la densidad ósea puede ser mantenida con dosis de estrógenos inclusive más bajas que las necesarias para inhibir la ovulación. Este aspecto de los AO requiere de todas maneras de más investigación antes de que se pueda llegar a alguna conclusión definitiva.

\section{Control del ciclo con la píldora de 20 ug de EE y 75 ug de GTD}

Para que este tipo de píldora sea ampliamente aceptada y utilizada, se debe contar con la tranquilidad de que su seguridad anticonceptiva no está afectada y que a la vez se mantiene un adecuado control del ciclo. Es decir que sigue siendo muy efectiva y bien tolerada.

En el estudio multicéntrico, abierto y a largo plazo más grande que se ha realizado (17), 670 mujeres de 18 a 45 años durante un período de 3 años con 19.095 ciclos disponibles para evaluación, se observó un adecuado control del ciclo en mujeres que no olvidaron tomar ninguna de las píldoras (95\% de ellas ). El ciclo normal antes del estudio osciló entre 21 a 28 días en $86.7 \%$ de las pacientes, conservandolo entre el 80 al $94 \%$ de las mujeres durante el estudio. No se observaron cambios en ningún momento en relación a la hemorragia por supresión con duración de 3 a 5 días, que la presentaron antes y durante el estudio el $83 \%$ de las participantes, $15.2 \%$ presentaban hemorragia por supresión mayor a 5 días, cifra que disminuyó al $10 \%$ a lo largo del estudio, siendo del $4.3 \%$ al final del mismo.

La intensidad de la menstruación no se vio mayormente afectada, $72.9 \%$ informaron que era normal, $22.7 \%$ que era ligera y $4.4 \%$ como fuerte, cifras que cambiaron sólo a $75.9 \%, 22.8 \%$ y $2.3 \%$ respectivamente.

La amenorrea fue mayor en el primer ciclo, $3.7 \%$ no presentaron hemorragia por supresión, $2.7 \%$ fue el máximo entre los ciclos 2 a 37, y la incidencia total fue de 122 mujeres presentándolo en 287 ciclos de un gran total de 19.095 ciclos, lo que da una cifra general del $1.5 \%$.

Lo que sí llama la atención es el comportamiento de la dismenorrea, la cual mejoró en 60 de 62 mujeres a lo largo del estudio, pero $8(1.3 \%)$ de las participantes desarrollaron esta molestia a lo largo de la investigación.

La tasa general de hemorragia intermenstrual calculada durante los 19.095 ciclos fue de $7.4 \%$, disminuyendo de $18.7 \%$ en el primer ciclo a $2.6 \%$ en el ciclo 36 . A1 final del estudio tan sólo $1 \%$ informaban de manchado y $1.6 \%$ de hemorragia por escape. Esto en general, sin tomar en cuenta las que incumplían con el tratamiento al olvidar y no tomar las píldoras, en quienes se presenta una correlación entre el cumplimiento y la presencia de hemorragia intermenstrual, $41.4 \%$ en el ciclo 1 de las olvidadizas vs. $17.7 \%$ de las que no se olvidaron de la píldora. Al final del ciclo 12 sólo $6.4 \%$ de las que cumplieron presentaron hemorragia intermenstrual, frente al $30.8 \%$ de las olvidadizas.

El perfil clínico de un Ao está influenciado no sólo por la dosis estrogénica sino también por el gestágeno presente en el preparado, y a lo largo de los años se ha reducido la dosis de los dos componentes, como ya se anotó antes. En relación a los preparados que contienen 30 / 35 ug de EE, varios estudios han demostrado una menor incidencia de manchado o de hemorragia intermenstrual por escape en los que poseen GTD -un gestágeno totalmente biodisponible- en comparación con aquellos que contienen una dosis idéntica de estrógeno combinado con el gestágeno DSG (18-20). Rosenberg (21) también elaboró un meta-análisis acerca del riesgo para hemorragia intermenstrual con los gestágenos modernos, y sugiere que existe un riesgo de $62 \%$ mayor de manchado o de hemorragia por escape con los anticonceptivos orales que contienen DSG y norgestimato (NGT) (combinados con 30 - 35 ug EE), al compararlos con el AO que contiene GTD.

Este menor riesgo para hemorragia intermenstrual de la píldora de 20 ug con GTD es confirmado también en el estudio comparativo con 20 ug EE - 75 ug GTD vs. $150 \mathrm{ug}$ DSG- de Short y Endrikat (22), en el cual se encuentra una tendencia a una menor incidencia de hemorragia intermenstrual en el grupo con GTD, aunque esta diferencia no fue significativa. Y en el caso del manchado, la incidencia acumulada durante los tres primeros ciclos que son los verdaderamente importantes para la aceptación por parte de la paciente- fue 3.5\% menor en el grupo con GTD, y durante los primeros 6 ciclos menor en $7.6 \%$ que en el grupo con DSG.

\section{Seguridad anticonceptiva}

Sólo una de 670 mujeres participantes en el estudio arriba mencionado (17) quedó embarazada, debido a su pobre cumplimiento. El diagnóstico se hizo durante el ciclo 34, con olvido de dos píldoras en el ciclo 31 y de una en el ciclo 32. El Indice de Pearl no corregido fue de 0.07 , resultado muy satisfactorio. La tasa de falla informada para el AO con 20 ug EE y 150 ug DSG es de 0.20 en el Indice de Pearl no corregido (2).

En un reciente estudio europeo, abierto, comparativo y multicéntrico (22) de 218 mujeres / 2495 ciclos en el grupo con GTD y 219 mujeres / 2496 ciclos en el grupo con DSG con 20 ug EE en ambos preparados, se presentaron en el grupo con GTD un embarazo debido a error en la ingesta y otro no relacionado con el estudio cuando la paciente interrumpió la toma de la píldora. En el grupo con DSG hubo dos embarazos, uno debido a error en la toma y otro a falla del método (Tabla 5).

\section{Cambios en el peso}

Durante el trabajo grande de investigación (17) se observó que al final del ciclo $1269.2 \%$ mantenían el 
mismo peso, aunque se anticipaba un aumento significativo debido a la naturaleza de largo plazo del estudio. Al final de ciclo $3654.8 \%$ mantenían su peso, 28.6\% habían aumentado por encima de $2 \mathrm{~kg}$ y $15.6 \%$ con una pérdida mayor a $2 \mathrm{~kg}$.

\section{La presión arterial}

No hubo cambios significativos durante el estudio anotado (17) en relación a la presión arterial.

\section{Conclusiones}

De acuerdo con todos los datos aquí presentados, el nuevo Ao de 20 ug EE y 75 ug GTD ha demostrado ser efectivo y bien tolerado. Con los argumentos clínicos y biológicos existentes hasta el momento, este preparado puede constituirse en un paso hacia adelante en el progreso y desarrollo de los Ao. Por lo tanto, su utilización en la anticoncepción oral, sobre todo a largo plazo, parece tener una justificación adecuada y ser recomendable. La reducción del estrógeno a 20 ug en el preparado con GTD no disminuye su eficacia, el control del ciclo sigue siendo bueno, ofreciendo la ventaja de reducir los efectos adversos relacionados con los estrógenos. Un AO con estas características es altamente deseable no sólo por parte de los médicos sino también por parte de sus pacientes.

\section{Recomendaciones}

Se debe ejercer precaución en el uso de AO combinados -ya sea que contengan gestágenos de última generación o alguno de los anteriores -para evitar su utilización en mujeres con antecedentes de problemas tromboembólicos personales o presentados durante un embarazo o familiares. En este campo -aunque el riesgo real es muy bajo- la precaución es aplicable sobre todo a las mujeres jóvenes -especialmente a las que nunca se han embarazado-, o a aquellas usuarias de primera vez nunca expuestas antes a los esteroides sexuales. Esto permitirá una mejor selección de las candidatas para AO y la prevención de problemas tromboembólicos mediante un elemento sencillo como el interrogatorio cuidadoso.

\section{BIBLIOGRAFIA}

1. Urdinola J., Gutiérrez A., Londoño D. Anticonceptivos orales en América Latina. Adv Contracep 1993; 9 (Suppl. 1): 3-12.

2. Urdinola J. Anticonceptivos orales: 30 vs. 20 ug de etinilestradiol (EE). Adv Contracept 1994; 10( Suppl.1): 41-49.

3. Rabe T., Runnebaum B. Arzneimittelpruefung von oralen Kontrazeptiva heute. En Hammerstein J (Ed): 25 Jahre hormonale Kontrazeptiva aus Berlin. Konsequente Dosisreduktion. Amsterdam, Excerpta Medica 1986; 62-72.

4. Inman WHW., Vessey MP. Investigation of deaths from pulmonary, coronary and cerebral thrombosis and embolism in Women of childbearing age. Br Med J 1968; 2: 193-199.

5. Cancer and Steroid Hormone Study of the Center for Disease Control and the National lnstitute of Child Health and Human Development: Combination oral contraceptive use and the risk of endometrial cancer. J Am Med Assoc 1987; 257: 796-780.

6. Cancer and Steroid Hormone Study of the Centers for Disease Control and the National Institute of Child Health and Human Development: The reduction in risk of ovarian cancer associated with oral contraceptive use. N Engl J Med 1987; 316: 650-655.

7. Rosenberg MJ., Waugh MS., Meehan TE. Use and misuse of oral contraceptives: risk indicators for poor pill taking and discontinuation. Contraception 1995; 51: 283-288.

8. Gerstman BB., Piper JM., Tomita DK., Ferguson WJ., Stadel BV., Lundin FE. Oral contraceptive estrogen dose and the risk of deep venous thromboembolic disease. Am J Epidemiol 1991; 133: 32-37.

9. Vandenbroucke JP., van der Meer FJM., Helmerhorst FM., Rosendaal F. Factor V Leiden: should we screen oral contraceptive users and pregnant women? BMJ 1996; 313: 1127-1130.

10. Mall-Haefeli M. Clinical experience with Mercilon and Marvelon with particular reference to ovarian function. Geburtsh Frauenheilk 1991; 51: 34-38.

11. Fitzergerald C., Feichtinger W., Spona J et al. A comparison of the effects of two monophasic low dose oral contraceptives on the inhibition of ovulation. Adv Contracept 1994; 10: 5-18.

12. Elomaa K., Moorees MT., De Prest J., Lahteenmaki PO. Extension of the pill-free period by three days in oral contraceptive users. Abstract 04.27- The XIV FIGO World Congress, Montreal, September 1994.

13. Spona J., Elstein M., Feichtinger W., Sullivan H., Lüdicke Fm Müller U, Düsterberg B: Shorter pill-free interval in combined oral contraceptives decreases follicular development. Contraception 1996 54: 71-77.
14. Cancer and Steroid Hormone Study of the Centers for Disease Control and the National lnstitute of Child Health and Human Development: Combination oral contraceptive use and the risk of endometrial cancer. JAMA 1987; 257: 796-800.

15. Cancer and Steroid Hormone Study of the Centers for Disease Control and the National lnstitute of child Health and Human Development: The reduction in risk of ovarian cancer associated with oral contraceptive use. JAMA 1987; 316: 650-655.

16. Polatti F., Perotti F., Filippa N., Gallina D., Nappi RE. Bone mass and long-term monophasic oral contraceptive treatment in young women. Contraception 1995; 51: 221-224.

17. Düsterberg B., Ellman H., Müller U., Rowe E., Mühe B. Three years'clinical experience with a new low-dose oral contraceptive containing 20 ug EE and 75 ug GTD: efficacy, cycle control and tolerability. En: Lopes Py Killick SR (Eds.): The New Option of LowDose Oral Contraception. Carnforth UK, The Parthenon Publishing Group 1996; 21-35.

18. Rosenberg MJ., Long SC. Oral contraceptives and cycle control. A critical review of the literature. Adv Contracept 1992; 8: (Suppl 1) 35.

19. Brill K., Müller C, Schnitker J., Albring M. The influence of different modern low-dose oral contraceptives on intermenstrual bleeding. Adv Contracept 1991; 7 (Suppl. 2): 51-61.

20. Latin American Oral Contraceptive Study Group: Clinical comparison of monophasic oral contraceptive preparations of gestodene / ethynilestradiol and desogestrel / ethynilestradiol. Contraception 1994; 50: 201-214.

21. Rosenberg MJ., Meehan T. The effect of desogestrel, gestodene; norgestimate and other factor on cycle control: a meta-analysis. Presentado en el Tercer Congreso de la Sociedad Europea de Anticoncepción, Dublin, 1994.

22. Short M., Endrikat J. A 12-month comparative investigation of reliability, cycle control and tolerance with low-dose oral contraceptives containing $20 \mathrm{ug}$ ethynilestradiol and either 75 ug gestodene or $150 \mathrm{ug}$ desogestrel. En: Lopes P y Killick SR (Eds.): The New Option of Low-Dose Oral Contraception. Carnforth UK, The Parthenon Publishing Group 1996; 37-47. 